Research Article

\title{
The Maximal ABC Index of the Corona of Two Graphs
}

\author{
Haiqin Liu $\mathbb{D}^{1,2}$ and Yanling Shao $\mathbb{D}^{3}$ \\ ${ }^{1}$ College of Big Data, North University of China, Taiyuan 030051, Shanxi, China \\ ${ }^{2}$ Basic Department, Shanxi Agricultural University, Taigu 030801, Shanxi, China \\ ${ }^{3}$ School of Science, North University of China, Taiyuan 030051, Shanxi, China \\ Correspondence should be addressed to Yanling Shao; ylshao@nuc.edu.cn
}

Received 11 November 2020; Revised 1 March 2021; Accepted 17 March 2021; Published 2 April 2021

Academic Editor: Bekir Sahin

Copyright (c) 2021 Haiqin Liu and Yanling Shao. This is an open access article distributed under the Creative Commons Attribution License, which permits unrestricted use, distribution, and reproduction in any medium, provided the original work is properly cited.

Let $G_{1} \circ G_{2}$ be the corona of two graphs $G_{1}$ and $G_{2}$ which is the graph obtained by taking one copy of $G_{1}$ and $\left|V\left(G_{1}\right)\right|$ copies of $G_{2}$ and then joining the $i$ th vertex of $G_{1}$ to every vertex in the $i$ th copy of $G_{2}$. The atom-bond connectivity index (ABC index) of a graph $G$ is defined as $A B C(G)=\sum_{u v \in E(G)} \sqrt{\left(d_{G}(u)+d_{G}(v)-2 / d_{G}(u) d_{G}(v)\right)}$, where $E(G)$ is the edge set of $G$ and $d_{G}(u)$ and $d_{G}(v)$ are degrees of vertices $u$ and $v$, respectively. For the ABC indices of $G_{1} \circ G_{2}$ with $G_{1}$ and $G_{2}$ being connected graphs, we get the following results. (1) Let $G_{1}$ and $G_{2}$ be connected graphs. The ABC index of $G_{1} \circ G_{2}$ attains the maximum value if and only if both $G_{1}$ and $G_{2}$ are complete graphs. If the ABC index of $G_{1} \circ G_{2}$ attains the minimum value, then $G_{1}$ and $G_{2}$ must be trees. (2) Let $T_{1}$ and $T_{2}$ be trees. Then, the ABC index of $T_{1} \circ T_{2}$ attains the maximum value if and only if $T_{1}$ is a path and $T_{2}$ is a star.

\section{Introduction}

Graph theory has been applied in many engineering fields such as mechanical design and manufacturing and chemical engineering. In the graph theory, the link in the mechanism can be regarded as the vertex, kinematic pair can be regarded as an edge, and then the topological configuration of the mechanism is abstracted as the graph. Therefore, the nature and characteristics of the mechanism can be analyzed by relevant graph theory. In chemical engineering, if a chemical molecule is regarded as a two-dimensional graph, the graph's vertices represent atoms, and edges represent chemical bonds, then the graph determines the topological properties of the given molecule.

Molecular descriptors play an important role in chemistry and pharmacology. Among these molecular descriptors, so-called topological indices play a significant role. Topological indices are the mathematical tools that correlate the chemical structure with various physical properties, chemical reactivity, or biological activity numerically. And the topological indices have been widely applied in the study of the stability of alkanes and the strain energy of cycloalkanes. In the field of pharmaceutical chemistry and bioinformatics, topological index can be used to encode the chemical structure. This encode strategy provides the annotation, comparison, rapid collection, mining, and retrieval of chemical structures within large databases. Afterward, topological indices can be used to look for quantitative structure-activity relationships and quantitative structureproperty relationships. In QSAR/QSPR studies, the biological activities of compounds can be predicted according to their topological indices, such as Zagreb, Randic, and the atom-bond connectivity indices.

The topological indices can be classified by the structural properties of graphs used for their calculation. The atombond connectivity index, which was proposed by Estrada et al. in 1998, is a vertex-degree-based graph topological index.

We consider finite undirected connected graphs without loops or multiple edges. Let $G_{1}$ and $G_{2}$ be two such graphs. The corona of $G_{1}$ and $G_{2}$, denoted by $G_{1} \circ G_{2}$, is defined as the graph obtained by taking one copy of $G_{1}$ and $\left|V\left(G_{1}\right)\right|$ copies of $G_{2}$ and then joining the $i$ th vertex of $G_{1}$ to every vertex in the $i$ th copy of $G_{2}$ (as shown in Figure 1). Corona graphs were introduced by Frucht and Harary in 1970 [1]. 

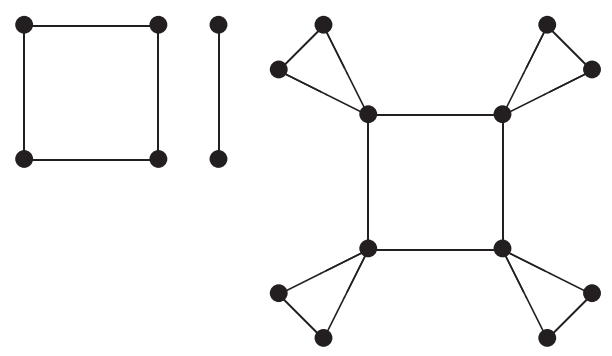

(a)

(b)

(c)

Figure 1: Corona $G_{1} \circ G_{2}$ of $G_{1}$ and $G_{2}$. (a) $G_{1}$. (b) $G_{2}$. (c) $G_{1} \circ G_{2}$.

Let $G=(V, E)$ be a connected graph with vertex set $V=$ $V(G)$ and $\operatorname{arc}$ set $E=E(G)$. The atom-bond connectivity index ( $A B C$ index) of $G$ is defined as

$$
A B C(G)=\sum_{u v \in E(G)} \sqrt{\frac{d_{G}(u)+d_{G}(v)-2}{d_{G}(u) d_{G}(v)}},
$$

where $d_{G}(u)\left(\right.$ or $\left.d_{u}\right)$ and $d_{G}(v)\left(\right.$ or $\left.d_{v}\right)$ are degrees of vertices $u$ and $v$, respectively.

Let $G$ be a graph. For an edge $e=u v \in E(G)$ and an edge set $E_{0} \subseteq E(G)$, denote

$$
\begin{aligned}
A B C_{G}(e) & =\sqrt{\frac{d(u)+d(v)-2}{d(u) d(v)},} \\
A B C_{G}\left(E_{0}\right) & =\sum_{e \in E_{0}} A B C_{G}(e) .
\end{aligned}
$$

We say that $A B C_{G}(e)$ is the $A B C$ index of $e$ and $A B C_{G}\left(E_{0}\right)$ is the $\mathrm{ABC}$ index of $E_{0}$.

Throughout the paper, we use $K_{n}, S_{n}$, and $P_{n}$ to denote the complete graph, the star, and the path of order $n$, respectively.

The ABC index attracted a lot of attention in the last few years. Several properties of $A B C$ index were established. In particular, if a new edge is inserted into $G$, then $A B C$ index necessarily increases (see Lemma 1).

Lemma 1 (see [2]). Let $\mathrm{G}$ be a simple graph with nonadjacent vertices $i$ and $j$. Then,

$$
A B C(G+\{i j\})>A B C(G) .
$$

It is evident that $K_{n}$ has the maximal $\mathrm{ABC}$ index, whereas the connected graph with the minimal $\mathrm{ABC}$ index must be a tree $($ see $[2,3])$. The smallest $A B C$ index of trees with $n$ pendant vertices was characterized in [4]. In contrast to the minimal case, the tree with the maximal $A B C$ index was easily identified as the star (see [5]). In [6], the maximum and minimum ABC indices of all unicyclic graphs and unicyclic chemical graphs were obtained, and the corresponding extremal graphs were also characterized. In $[7,8]$, the maximum values of the $\mathrm{ABC}$ indices in the class of all $n$-vertex bicyclic and tricyclic graphs were presented, respectively.

Recently, some researchers have paid more attention to the $A B C$ spectral radius which is associated with $A B C$ energy.
Chen [9] characterized the graphs with extremal ABC spectral radius for a class of given graphs. Lin et al. [10] determined the trees with the third, fourth, and fifth largest $A B C$ spectral radii.

For corona graph, Bian et al. [11] considered some Wienertype indices of the corona graphs. Lu and Xue [12] studied the Kirchhoff index of two corona graphs. In [13], the lower and upper bounds for $\mathrm{ABC}$ indices of edge corona product of graphs were given. In [14], the extremal edge-version $\mathrm{ABC}$ index of some graph operations was given. For more information, see [15-19].

Motivated by this, it is interesting to determine the extremal graphs among the set $\left\{G_{1} \circ G_{2} \mid G_{1}\right.$ and $G_{2}$ are connected graphs (trees) $\}$. From Lemma 1, the problem is simple if $G_{1}$ and $G_{2}$ are connected graphs, so we give some conclusions directly. We mainly consider the problem for the case that $G_{1}$ and $G_{2}$ are trees. The main results are as follows.

(1) Let $G_{1}$ and $G_{2}$ be connected graphs. The ABC index of $G_{1} \circ G_{2}$ attains the maximum value if and only if both $G_{1}$ and $G_{2}$ are complete graphs. If the $A B C$ index of $G_{1} \circ G_{2}$ attains the minimum value, then $G_{1}$ and $G_{2}$ must be trees.

(2) Let $T_{1}$ and $T_{2}$ be trees. The $A B C$ index of $T_{1} \circ T_{2}$ attains the maximum value if and only if $T_{1}$ is a path and $T_{2}$ is a star.

\section{Preliminaries}

The following lemmas are known somehow (for example, see $[7,8])$.

Lemma 2. Let $f(x, y)=\sqrt{(x+y-2 / x y)}$ with $x, y>1$. Then, $f(x, y)$ strictly decreases with $x$ for fixed $y \geq 2$, and the function $\partial f / \partial x$ increases with $x$. Similarly, the function $f(x, y)$ strictly decreases with $y$ for fixed $x \geq 2$, and the function $\partial f / \partial y$ increases with $y$.

Lemma 3. If $G$ is a graph with $\delta(G) \geq 2(\delta(G)$ is the minimum degree of $G)$, then for any $x y \in E(G)$, $\sqrt{\left(d_{x}+d_{y}-2 / d_{x} d_{y}\right)} \leq(\sqrt{2} / 2)$. Equality holds if and only if $d_{x}=2$ or $d_{y}=2$.

Lemma 4. Let $f(x, y)=\sqrt{(x+y-2 / x y)}$ with $x, y>1$ and $g(x, y, z)=f(x, y)+f(z, y)-f(x+1, y)-f(z-1, y)$.

$$
\text { If } x \geq z \geq 2 \text {, then } g(x, y, z)<0 \text {. }
$$




\section{The Bounds of ABC Indices of $G_{1} \circ G_{2}$}

Let $G_{1}$ and $G_{2}$ be two simple connected graphs of orders $n_{1}$ and $n_{2}$, respectively, and $G=G_{1} \circ G_{2}$. For a vertex $v \in V\left(G_{1}\right)$, we use $G_{2}^{v}$ to denote the copy of $G_{2}$ in $G$ which attaches to the vertex $v$ of $G_{1}$. Denote

$$
\begin{aligned}
& E_{1}(G)=E\left(G_{1}\right), \\
& E_{2}(G)=\cup_{v \in V\left(G_{1}\right)}\left\{v u \mid u \in V\left(G_{2}^{v}\right)\right\}, \\
& E_{3}(G)=\cup_{v \in V\left(G_{1}\right)} E\left(G_{2}^{v}\right) .
\end{aligned}
$$

Then, the following four conditions hold:

(C1) $V(G)=V\left(G_{1}\right) \cup\left(\cup_{v \in V\left(G_{1}\right)} V\left(G_{2}^{v}\right)\right)$.

(C2) $\quad E(G)=E_{1}(G) \cup E_{2}(G) \cup E_{3}(G)$,

and

$E_{k}(G) \cap E_{l}(G)=\phi$ for $k, l=1,2,3$ and $k \neq l$.

(C3) For $v \in V\left(G_{1}\right)$ and $u \in V\left(G_{2}^{v}\right), d_{G}(v)=d_{G_{1}}(v)+$ $n_{2}$ and $d_{G}(u)=d_{G_{2}}(u)+1$.

(C4) $A B C(G)=A B C_{G}\left(E_{1}(G)\right)+A B C_{G}\left(E_{2}(G)\right)+A B$ $C_{G}\left(E_{3}(G)\right)$.

From (C4) and (C3), the following theorem is obtained immediately, and we omit the proof.

Theorem 1. Let $G_{1}$ and $G_{2}$ be two simple connected graphs of orders $n_{1}$ and $n_{2}$, respectively, and $G=G_{1} \circ G_{2}$. Then,

$$
\begin{aligned}
A B C(G)= & \sum_{u v \in E\left(G_{1}\right)} \sqrt{\frac{d_{G_{1}}(u)+d_{G_{1}}(v)+2 n_{2}-2}{\left(d_{G_{1}}(u)+n_{2}\right)\left(d_{G_{1}}(v)+n_{2}\right)}} \\
& +\sum_{v \in V\left(G_{1}\right), u \in\left(G_{2}^{v}\right)} \sqrt{\frac{d_{G_{1}}(v)+d_{G_{2}}(u)+n_{2}-1}{\left(d_{G_{1}}(v)+n_{2}\right)\left(d_{G_{2}}(u)+1\right)}} \\
& +n_{1} \sum_{u v \in E\left(G_{2}\right)} \sqrt{\frac{d_{G_{2}}(u)+d_{G_{2}}(v)}{\left(d_{G_{2}}(u)+1\right)\left(d_{G_{2}}(v)+1\right)}}
\end{aligned}
$$
clear.

By Lemma 1 and Theorem 1, the following results are

Lemma 5. Let $G_{1}$ and $G_{2}$ be two simple connected graphs of orders $n_{1} \geq 3$ and $n_{2} \geq 3$, respectively. Then, for any edge $x_{1} x_{2} \in E\left(G_{1}\right)$,

$$
A B C\left(G_{1} \circ G_{2}\right)>A B C\left[\left(G_{1}-x_{1} x_{2}\right) \circ G_{2}\right] .
$$

Lemma 6. Let $G_{1}$ and $G_{2}$ be two simple connected graphs of orders $n_{1} \geq 3$ and $n_{2} \geq 3$, respectively. Then, for any edge $x_{1} x_{2} \in E\left(G_{2}\right)$,

$$
A B C\left(G_{1} \circ G_{2}\right)>A B C\left[G_{1} \circ\left(G_{2}-x_{1} x_{2}\right)\right] .
$$

Theorem 2. Let $G_{1}$ and $G_{2}$ be two simple connected graphs of orders $n_{1} \geq 3$ and $n_{2} \geq 3$, respectively. Then,

$$
\begin{aligned}
A B C\left(G_{1} \circ G_{2}\right) \leq & A B C\left(K_{n_{1}} \circ K_{n_{2}}\right) \\
= & \frac{n_{1}\left(n_{1}-1\right)}{2} \frac{\sqrt{2\left(n_{1}+n_{2}-1\right)-2}}{n_{1}+n_{2}-1} \\
& +n_{1} n_{2} \sqrt{\frac{n_{1}+2 n_{2}-3}{n_{2}\left(n_{1}+n_{2}-1\right)}}
\end{aligned}
$$

$$
+\frac{n_{1} n_{2}\left(n_{2}-1\right)}{2} \frac{\sqrt{2 n_{2}-2}}{n_{2}},
$$

and the equality holds if and only if $G_{1}=K_{n_{1}}$ and $G_{2}=K_{n_{2}}$.

Lemmas 5 and 6 show that deleting an edge in one of the graphs $\left(G_{1}\right.$ and $\left.G_{2}\right)$ will decrease the ABC index of $G_{1} \circ G_{2}$. Consequently, we get the necessary condition for the $\mathrm{ABC}$ indices of $G_{1} \circ G_{2}$ to be the minimum.

Theorem 3. If the $A B C$ index of $G_{1} \circ G_{2}$ attains the minimum value, then $G_{1}$ and $G_{2}$ must be trees.

\section{The Upper Bound of $\mathrm{ABC}$ Indices of $T_{1}{ }^{\circ} T_{2}$}

Since the minimal $A B C$ trees are not unique $($ see $[2,5])$, it seems to be difficult to characterize the corona of two trees with minimal $A B C$ index. We leave the problem as a future task. In this section, we are going to give the upper bound of $\mathrm{ABC}$ indices for $T_{1} \circ T_{2}$.

Lemma 7. Let $T_{1}$ be any tree of order $n_{1} \geq 3$ and $T_{2}$ be a tree of order $n_{2} \geq 4$ as depicted in Figure 2, where $u_{1 i}$ and $u_{2 j}$ are pendant vertices for $i=1, \ldots, s$ and $j=1, \ldots, t$ with $s \geq 1$, $t \geq 1, \quad u_{1} \neq u_{2}, \quad$ and $\quad d_{T_{2}}\left(u_{2}\right) \geq d_{T_{2}}\left(u_{1}\right)$. Let $T_{3}=T_{2}-$ $u_{1} u_{1 s}+u_{2} u_{1 s}$. Then,

$$
A B C\left(E_{2}\left(T_{1} \circ T_{2}\right)\right)<A B C\left(E_{2}\left(T_{1} \circ T_{3}\right)\right) .
$$

Proof. Denote $T=T_{1} \circ T_{2}$, and $T^{\prime}=T_{1} \circ T_{3}$. Let $v$ be any vertex of $T_{1}$. We can get the result if we prove that

$$
\sum_{u \in V\left(T_{2}^{v}\right)} A B C_{T}(v u)<\sum_{u \in V\left(T_{3}^{v}\right)} A B C_{T^{\prime}}(v u)
$$

Note that both trees $T_{2}$ and $T_{3}$ have the same vertex set, and except $u_{1}$ and $u_{2}$, the other vertices have the same edges in $T_{2}$ and $T_{3}$. So, 


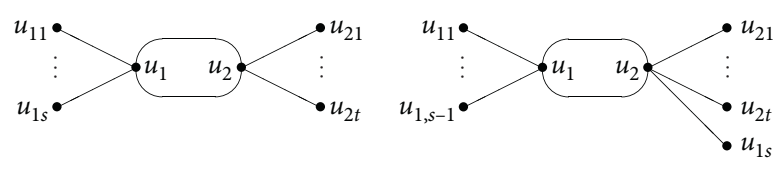

(a)

(b)

Figure 2: Tress $T_{2}$ and $T_{3}$. (a) Tree $T_{2}$. (b) Tree $T_{3}$.

$$
\begin{gathered}
\sum_{u \in V\left(T_{2}^{v}\right)} A B C_{T}(v u)-\sum_{u \in V\left(T_{3}^{v}\right)} A B C_{T^{\prime}}(v u) \\
=\sqrt{\frac{\left(d_{T_{1}}(v)+n_{2}\right)+\left(d_{T_{2}}\left(u_{1}\right)+1\right)-2}{\left(d_{T_{1}}(v)+n_{2}\right)\left(d_{T_{2}}\left(u_{1}\right)+1\right)}} \\
+\sqrt{\frac{\left(d_{T_{1}}(v)+n_{2}\right)+\left(d_{T_{2}}\left(u_{2}\right)+1\right)-2}{\left(d_{T_{1}}(v)+n_{2}\right)\left(d_{T_{2}}\left(u_{2}\right)+1\right)}} \\
-\sqrt{\frac{\left(d_{T_{1}}(v)+n_{2}\right)+\left(d_{T_{3}}\left(u_{1}\right)+1\right)-2}{\left(d_{T_{1}}(v)+n_{2}\right)\left(d_{T_{3}}\left(u_{1}\right)+1\right)}} \\
-\sqrt{\frac{\left(d_{T_{1}}(v)+n_{2}\right)+\left(d_{T_{3}}\left(u_{2}\right)+1\right)-2}{\left(d_{T_{1}}(v)+n_{2}\right)\left(d_{T_{3}}\left(u_{2}\right)+1\right)}}
\end{gathered}
$$

Note that $d_{T_{3}}\left(u_{1}\right)=d_{T_{2}}\left(u_{1}\right)-1, d_{T_{3}}\left(u_{2}\right)=d_{T_{2}}\left(u_{2}\right)+1$, and $d_{T_{2}}\left(u_{2}\right) \geq d_{T_{2}}\left(u_{1}\right) \geq 2$. Then, by Lemma 4 (taking $x=d_{T_{2}}\left(u_{2}\right)+1, y=d_{T_{1}}(v)+n_{2}$, and $\left.z=d_{T_{2}}\left(u_{1}\right)+1\right)$,

$$
\sum_{u \in V\left(T_{2}^{v}\right)} A B C_{T}(v u)-\sum_{u \in V\left(T_{3}^{v}\right)} A B C_{T^{\prime}}(v u)<0 .
$$

The lemma holds.

Using Lemma 7 repeatedly, we can obtain the following result.

Corollary 1. Let $T_{1}$ and $T_{2}$ be two trees of orders $n_{1} \geq 3$ and $n_{2} \geq 3$, respectively. Then,

$$
A B C_{T}\left(E_{2}\left(T_{1} \circ T_{2}\right)\right) \leq A B C_{T^{\prime}}\left(E_{2}\left(T_{1} \circ S_{n_{2}}\right)\right),
$$

and the equality holds if and only if $T_{2}=S_{n_{2}}$.
Theorem 4. Let $T_{1}$ and $T_{2}$ be two trees of orders $n_{1} \geq 3$ and $n_{2} \geq 3$, respectively. Then,

$$
A B C\left(T_{1} \circ T_{2}\right) \leq A B C\left(T_{1} \circ S_{n_{2}}\right),
$$

and the equality holds if and only if $T_{2}=S_{n_{2}}$.

Proof. Denote $T=T_{1} \circ T_{2}$ and $T^{\prime}=T_{1} \circ S_{n_{2}}$. Note $A B C_{T}\left(E_{1}(T)\right)=A B C_{T^{\prime}}\left(E_{1}\left(T^{\prime}\right)\right)$. By Corollary 1 , we can get the result if we show $A B C_{T}\left(E_{3}(T)\right) \leq A B C_{T^{\prime}}\left(E_{3}\left(T^{\prime}\right)\right)$.

Note that

$$
\begin{aligned}
A B C_{T}\left(E_{3}(T)\right) & =\sum_{u v \in E\left(T_{2}\right)} \sqrt{\frac{\left(d_{T_{2}}(u)+1\right)+\left(d_{T_{2}}(v)+1\right)-2}{\left(d_{T_{2}}(u)+1\right)\left(d_{T_{2}}(v)+1\right)}} \\
A B C_{T^{\prime}}\left(E_{3}\left(T^{\prime}\right)\right) & =\sum_{u v \in E\left(S_{n_{2}}\right)} \sqrt{\frac{\left(d_{S_{n_{2}}}(u)+1\right)+\left(d_{S_{n_{2}}}(v)+1\right)-2}{\left(d_{S_{n_{2}}}(u)+1\right)\left(d_{S_{n_{2}}}(v)+1\right)}} \\
& =\left(n_{2}-1\right) \sqrt{\frac{1}{2} .}
\end{aligned}
$$

By Lemma 3,

$$
A B C_{T}\left(E_{3}(T)\right) \leq \sum_{u v \in E\left(T_{2}\right)} \sqrt{\frac{1}{2}}=\left(n_{2}-1\right) \sqrt{\frac{1}{2}}=A B C_{T^{\prime}}\left(E_{3}\left(T^{\prime}\right)\right)
$$

and equality holds if and only if $d_{T_{2}}(u)+1=2$ or $d_{T_{2}}(v)+$ $1=2$ for each edge $u v \in E\left(T_{2}\right)$, that is, $T_{2}=S_{n_{2}}$. The result follows now.

Theorem 5. Let $T_{1}$ and $T_{1}^{\prime}$ be two trees as depicted in Figure 3, which contain $T_{0}$ as a subtree, where $d_{v}=d_{T_{1}}(v) \geq 3$, and $l \geq k \geq 1$. Let $n_{2} \geq 4$. Then, 

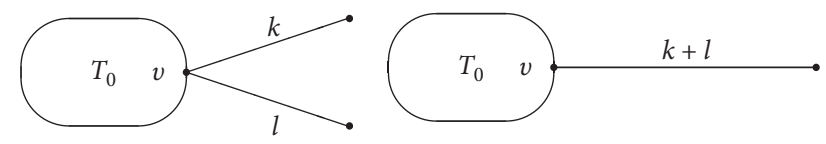

(a)

(b)
Figure 3: Trees $T_{1}$ and $T_{1}^{\prime}$. (a) Tree $T_{1}$. (b) Tree $T_{1}^{\prime}$.

$$
A B C\left(T_{1} \circ S_{n_{2}}\right)<A B C\left(T_{1}^{\prime} \circ S_{n_{2}}\right) .
$$

Proof. Denote $T=T_{1} \circ S_{n_{2}}$ and $T^{\prime}=T_{1}^{\prime} \circ S_{n_{2}}$. Note that $A B C_{T}\left(E_{3}(T)\right)=A B C_{T^{\prime}}\left(E_{3}\left(T^{\prime}\right)\right)$. We can get the result if we show

$A B C_{T}\left(E_{1}(T)\right)+A B C_{T}\left(E_{2}(T)\right)<A B C_{T^{\prime}}\left(E_{1}\left(T^{\prime}\right)\right)+A B C_{T^{\prime}}\left(E_{2}\left(T^{\prime}\right)\right)$.

Firstly, we consider $A B C_{T}\left(E_{2}(T)\right)-A B C_{T^{\prime}}\left(E_{2}\left(T^{\prime}\right)\right)$. Let $u$ be any vertex of $T_{1}\left(T_{1}^{\prime}\right)$. If $w \in V\left(S_{n_{2}}\right)$ is a pendant vertex of $S_{n_{2}}$, then the ABC index of $u w$ is $\sqrt{1 / 2}$. If $u$ is a pendant vertex of $T_{1}$ and $T_{1}^{\prime}$ and $w \in V\left(S_{n_{2}}\right)$ is the central vertex of $S_{n_{2}}$, then the ABC index of $u w$ has no change. So, we only need to calculate the $\mathrm{ABC}$ index of $u w$, where $u$ is not a pendant vertex of $T_{1}^{\prime}$ and $w$ is the central vertex of $S_{n_{2}}$.

Denote $a=n_{2}+2, b=d_{v}+n_{2}, \quad A=N_{T_{0}}(v)$. Then, $b>a \geq 6$ and

$$
\begin{aligned}
& A B C_{T}\left(E_{2}(T)\right)-A B C_{T^{\prime}}\left(E_{2}\left(T^{\prime}\right)\right) \\
& =\sqrt{\frac{\left(d_{v}+n_{2}\right)+n_{2}-2}{\left(d_{v}+n_{2}\right) n_{2}}+\sqrt{\frac{\left(1+n_{2}\right)+n_{2}-2}{\left(1+n_{2}\right) n_{2}}}} \\
& -\sqrt{\frac{\left(d_{v}+n_{2}-1\right)+n_{2}-2}{\left(d_{v}+n_{2}-1\right) n_{2}}}-\sqrt{\frac{\left(2+n_{2}\right)+n_{2}-2}{\left(2+n_{2}\right) n_{2}}} \\
& =\sqrt{\frac{a+b-4}{b(a-2)}}+\sqrt{\frac{2 a-5}{(a-1)(a-2)}}-\sqrt{\frac{a+b-5}{(b-1)(a-2)}}-\sqrt{\frac{2}{a}} .
\end{aligned}
$$

Combining the situation of $A B C_{T}\left(E_{1}(T)\right)-A B C_{T^{\prime}}\left(E_{1}\left(T^{\prime}\right)\right)$, we consider the following three cases.

Case 1. $k=l=1$.

By Lemma 2,

$$
\begin{aligned}
& A B C_{T}\left(E_{1}(T)\right)-A B C_{T^{\prime}}\left(E_{1}\left(T^{\prime}\right)\right) \\
& =2 \sqrt{\frac{\left(1+n_{2}\right)+\left(d_{v}+n_{2}\right)-2}{\left(1+n_{2}\right)\left(d_{v}+n_{2}\right)}}
\end{aligned}
$$$$
+\sum_{x \in A} \sqrt{\frac{\left(d_{x}+n_{2}\right)+\left(d_{v}+n_{2}\right)-2}{\left(d_{x}+n_{2}\right)\left(d_{v}+n_{2}\right)}}
$$$$
-\sqrt{\frac{\left(2+n_{2}\right)+\left(d_{v}+n_{2}-1\right)-2}{\left(2+n_{2}\right)\left(d_{v}+n_{2}-1\right)}}
$$$$
-\sqrt{\frac{\left(1+n_{2}\right)+\left(2+n_{2}\right)-2}{\left(1+n_{2}\right)\left(2+n_{2}\right)}}
$$$$
-\sum_{x \in A} \sqrt{\frac{\left(d_{x}+n_{2}\right)+\left(d_{v}+n_{2}-1\right)-2}{\left(d_{x}+n_{2}\right)\left(d_{v}+n_{2}-1\right)}}
$$$$
<2 \sqrt{\frac{\left(1+n_{2}\right)+\left(d_{v}+n_{2}\right)-2}{\left(1+n_{2}\right)\left(d_{v}+n_{2}\right)}}
$$$$
-\sqrt{\frac{\left(2+n_{2}\right)+\left(d_{v}+n_{2}-1\right)-2}{\left(2+n_{2}\right)\left(d_{v}+n_{2}-1\right)}}
$$

$$
-\sqrt{\frac{\left(1+n_{2}\right)+\left(2+n_{2}\right)-2}{\left(1+n_{2}\right)\left(2+n_{2}\right)}}
$$

$=2 \sqrt{\frac{a+b-3}{b(a-1)}}-\sqrt{\frac{a+b-3}{a(b-1)}}-\sqrt{\frac{2 a-3}{a(a-1)}}$,

$A B C_{T}\left(E_{1}(T)\right)+A B C_{T}\left(E_{2}(T)\right)-A B C_{T^{\prime}}\left(E_{1}\left(T^{\prime}\right)\right)$

$-A B C_{T^{\prime}}\left(E_{2}\left(T^{\prime}\right)\right)$

$<2 \sqrt{\frac{a+b-3}{b(a-1)}}-\sqrt{\frac{a+b-3}{a(b-1)}}-\sqrt{\frac{2 a-3}{a(a-1)}}$

$+\sqrt{\frac{a+b-4}{b(a-2)}}+\sqrt{\frac{2 a-5}{(a-1)(a-2)}}$

$-\sqrt{\frac{a+b-5}{(b-1)(a-2)}}-\sqrt{\frac{2}{a}}$

$\triangleq f_{1}(a, b)$ 
The function $f_{1}(a, b)$ has the following properties :

(1) The function $f_{1}(a, b)$ is a strictly decreasing function on $b$ for fixed $a \geq 6$.

We can see the result from the graph of $f_{1}(a, b)$. For example, the graphs of $f_{1}(6, b)$ and $f_{1}(10, b)$ are as follows (as depicted in Figures 4 and 5).

So,

$$
\begin{aligned}
f_{1}(a, b) \leq & f_{1}(a, a+1) \\
& =2 \sqrt{\frac{2}{a+1}}-\sqrt{\frac{2 a-2}{a^{2}}}-\sqrt{\frac{2 a-3}{a(a-1)}} \\
& +\sqrt{\frac{2 a-3}{(a+1)(a-2)}}+\sqrt{\frac{2 a-5}{(a-1)(a-2)}}-2 \sqrt{\frac{2}{a}}
\end{aligned}
$$

(2) The maximum value of the function $f_{1}(a, a+1)$ is attained when $a$ is sufficiently large.

We can get this result from the graph (as depicted in Figure 6) of $f_{1}(a, a+1)$.

Then,

$$
f_{1}(a, a+1)<\lim _{a \longrightarrow+\infty} f_{1}(a, a+1)=0 .
$$

So, for $b>a \geq 6, f_{1}(a, b)<0$ and the theorem follows for Case 1.

Case 2. $k=1$ and $l \geq 2$.

By Lemma 2,

$$
\begin{aligned}
& A B C_{T}\left(E_{1}(T)\right)-A B C_{T^{\prime}}\left(E_{1}\left(T^{\prime}\right)\right) \\
& =\sqrt{\frac{\left(d_{v}+n_{2}\right)+\left(1+n_{2}\right)-2}{\left(d_{v}+n_{2}\right)\left(1+n_{2}\right)}}+\sqrt{\frac{\left(d_{v}+n_{2}\right)+\left(2+n_{2}\right)-2}{\left(d_{v}+n_{2}\right)\left(2+n_{2}\right)}} \\
& +\sum_{x \in A} \sqrt{\frac{\left(d_{v}+n_{2}\right)+\left(d_{x}+n_{2}\right)-2}{\left(d_{v}+n_{2}\right)\left(d_{x}+n_{2}\right)}} \\
& -\sqrt{\frac{\left(d_{v}+n_{2}-1\right)+\left(2+n_{2}\right)-2}{\left(d_{v}+n_{2}-1\right)\left(2+n_{2}\right)}}-\sqrt{\frac{\left(2+n_{2}\right)+\left(2+n_{2}\right)-2}{\left(2+n_{2}\right)\left(2+n_{2}\right)}} \\
& -\sum_{x \in A} \sqrt{\frac{\left(d_{v}+n_{2}-1\right)+\left(d_{x}+n_{2}\right)-2}{\left(d_{v}+n_{2}-1\right)\left(d_{x}+n_{2}\right)}} \\
& <\sqrt{\frac{\left(d_{v}+n_{2}\right)+\left(1+n_{2}\right)-2}{\left(d_{v}+n_{2}\right)\left(1+n_{2}\right)}}+\sqrt{\frac{\left(d_{v}+n_{2}\right)+\left(2+n_{2}\right)-2}{\left(d_{v}+n_{2}\right)\left(2+n_{2}\right)}} \\
& -\sqrt{\frac{\left(d_{v}+n_{2}-1\right)+\left(2+n_{2}\right)-2}{\left(d_{v}+n_{2}-1\right)\left(2+n_{2}\right)}}-\sqrt{\frac{\left(2+n_{2}\right)+\left(2+n_{2}\right)-2}{\left(2+n_{2}\right)\left(2+n_{2}\right)}} \\
& =\sqrt{\frac{a+b-3}{b(a-1)}}+\sqrt{\frac{a+b-2}{a b}}-\sqrt{\frac{a+b-3}{a(b-1)}}-\sqrt{\frac{2 a-2}{a^{2}}}, \\
& A B C_{T}\left(E_{1}(T)\right)+A B C_{T}\left(E_{2}(T)\right)-A B C_{T^{\prime}}\left(E_{1}\left(T^{\prime}\right)\right) \\
& -A B C_{T^{\prime}}\left(E_{2}\left(T^{\prime}\right)\right) \\
& <\sqrt{\frac{a+b-3}{b(a-1)}}+\sqrt{\frac{a+b-2}{a b}}-\sqrt{\frac{a+b-3}{a(b-1)}}-\sqrt{\frac{2 a-2}{a^{2}}} \\
& +\sqrt{\frac{a+b-4}{b(a-2)}}+\sqrt{\frac{2 a-5}{(a-1)(a-2)}}-\sqrt{\frac{a+b-5}{(b-1)(a-2)}}-\sqrt{\frac{2}{a}} \\
& \triangleq f_{2}(a, b) \text {. }
\end{aligned}
$$




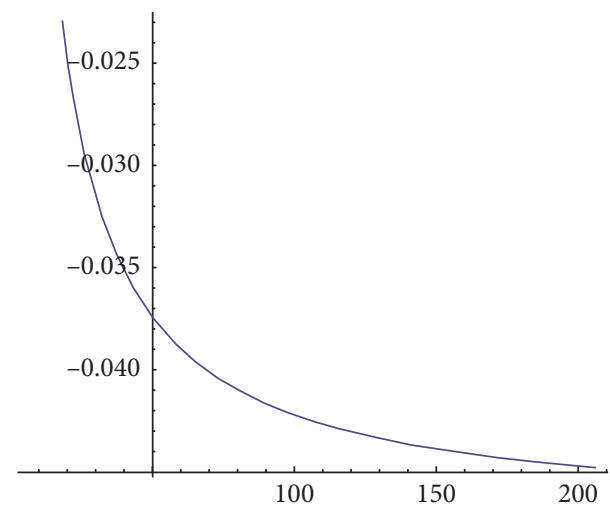

Figure 4: $f_{1}(6, b)$ with $b>6$.

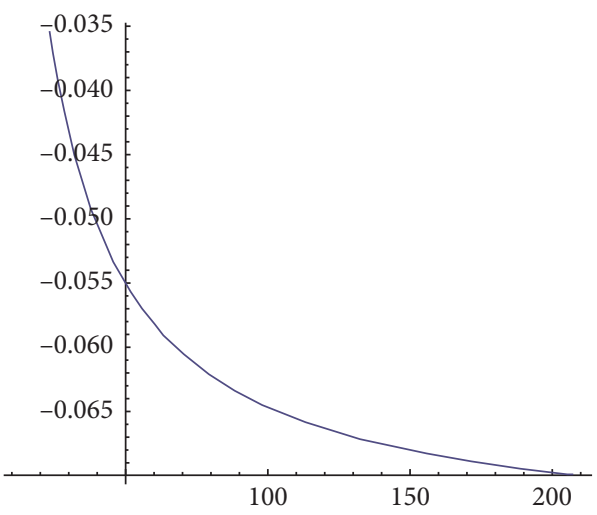

Figure 5: $f_{1}(10, b)$ with $b>10$.

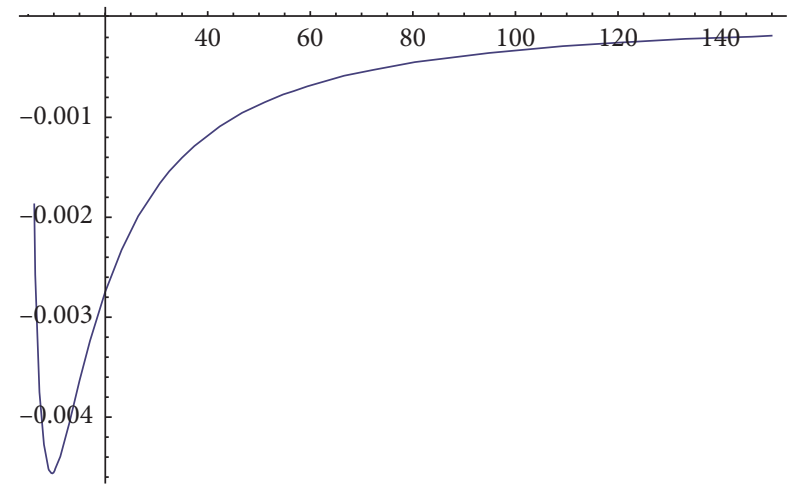

Figure 6: $f_{1}(a, a+1)$.

Similar to the discussions in Case 1 , we have that the function $f_{2}(a, b)$ is a strictly decreasing function on $b$ for fixed $a \geq 6$, and the maximum value of the function $f_{2}(a, a+$ 1 ) is attained when $a$ is sufficiently large. So,

$$
f_{2}(a, b) \leq f_{2}(a, a+1)<\lim _{a \longrightarrow+\infty} f_{2}(a, a+1)=0 .
$$

The theorem holds for Case 2 .

Case 3. $l \geq k \geq 2$.

By Lemma 2,

$$
\begin{array}{rl}
A & A C_{T}\left(E_{1}(T)\right)-A B C_{T^{\prime}}\left(E_{1}\left(T^{\prime}\right)\right) \\
= & 2 \sqrt{\frac{\left(d_{v}+n_{2}\right)+\left(2+n_{2}\right)-2}{\left(d_{v}+n_{2}\right)\left(2+n_{2}\right)}+\sqrt{\frac{\left(2+n_{2}\right)+\left(1+n_{2}\right)-2}{\left(2+n_{2}\right)\left(1+n_{2}\right)}}} \\
& +\sum_{x \in A} \sqrt{\frac{\left(d_{v}+n_{2}\right)+\left(d_{x}+n_{2}\right)-2}{\left(d_{v}+n_{2}\right)\left(d_{x}+n_{2}\right)}} \\
& -\sqrt{\frac{\left(d_{v}+n_{2}-1\right)+\left(2+n_{2}\right)-2}{\left(d_{v}+n_{2}-1\right)\left(2+n_{2}\right)}}-2 \sqrt{\frac{\left(2+n_{2}\right)+\left(2+n_{2}\right)-2}{\left(2+n_{2}\right)\left(2+n_{2}\right)}} \\
& -\sum_{x \in A} \sqrt{\frac{\left(d_{v}+n_{2}-1\right)+\left(d_{x}+n_{2}\right)-2}{\left(d_{v}+n_{2}-1\right)\left(d_{x}+n_{2}\right)}} \\
< & \sqrt{\frac{\left(d_{v}+n_{2}\right)+\left(2+n_{2}\right)-2}{\left(d_{v}+n_{2}\right)\left(2+n_{2}\right)}+\sqrt{\frac{\left(2+n_{2}\right)+\left(1+n_{2}\right)-2}{\left(2+n_{2}\right)\left(1+n_{2}\right)}}} \\
& -\sqrt{\frac{\left(d_{v}+n_{2}-1\right)+\left(2+n_{2}\right)-2}{\left(d_{v}+n_{2}-1\right)\left(2+n_{2}\right)}-2 \sqrt{\frac{\left(2+n_{2}\right)+\left(2+n_{2}\right)-2}{\left(2+n_{2}\right)\left(2+n_{2}\right)}}} \\
= & 2 \sqrt{\frac{a+b-2}{a b}+\sqrt{\frac{2 a-3}{a(a-1)}}-\sqrt{\frac{a+b-3}{a(b-1)}}-2 \sqrt{\frac{2 a-2}{a^{2}}}} \\
\triangleq & f_{3}(a, b) . \\
A B C_{T}\left(E_{1}(T)\right)+A B C_{T}\left(E_{2}(T)\right)-A B C_{T^{\prime}}\left(E_{1}\left(T^{\prime}\right)\right) \\
& -A B C_{T^{\prime}}\left(E_{2}\left(T^{\prime}\right)\right) \\
<2 & \sqrt{\frac{a+b-2}{a b}+\sqrt{\frac{a+b-4}{a(a-2)}}+\sqrt{\frac{2 a-3}{(a-1)(a-2)}}-\sqrt{\frac{a+b}{(b-1)(a-2)}}-\sqrt{\frac{a+b-3}{a(b-1)}}-2 \sqrt{\frac{2 a-2}{a^{2}}}}
\end{array}
$$

Similar to the discussions in Case 1, we have that the function $f_{3}(a, b)$ is a strictly decreasing function on $b$ for fixed $a \geq 6$, and the maximum value of the function $f_{3}(a, a+$ 1 ) is attained when $a$ is sufficiently large. So,

$$
f_{3}(a, b) \leq f_{3}(a, a+1)<\lim _{a \longrightarrow+\infty} f_{3}(a, a+1)=0 .
$$

Then, the theorem holds for Case 3. 
By Theorems 4, 5, and 1, we get the main result of this section.

Theorem 6. Let $T_{1}$ and $T_{2}$ be trees of orders $n_{1} \geq 3$ and $n_{2} \geq 4$, respectively. Then,

$$
\begin{aligned}
A B C\left(T_{1} \circ T_{2}\right) \leq & A B C\left(P_{n_{1}} \circ S_{n_{2}}\right)=2 \sqrt{\frac{2 n_{2}+1}{\left(n_{2}+1\right)\left(n_{2}+2\right)}} \\
& +\left(n_{1}-3\right) \sqrt{\frac{2 n_{2}+2}{\left(n_{2}+2\right)\left(n_{2}+2\right)}} \\
& +2 \sqrt{\frac{2 n_{2}-1}{n_{2}\left(n_{2}+1\right)}} \\
& +\left(n_{1}-2\right) \sqrt{\frac{2}{n_{2}+2}}+2 n_{1}\left(n_{2}-1\right) \sqrt{\frac{1}{2}}
\end{aligned}
$$

and the equality holds if and only if $T_{1}=P_{n_{1}}$ and $T_{2}=S_{n_{2}}$.

The values of $\mathrm{ABC}$ indices of $T_{1}{ }^{\circ} \mathrm{T}_{2}$ for $n_{1}<3, n_{2}<4$ are as follows.

Theorem 7. Let $T_{1}$ and $T_{2}$ be trees of orders $n_{1}<3$ and $n_{2}<4$, respectively. Then,

(1) $n_{1}=1, n_{2}=1, A B C\left(T_{1} \circ T_{2}\right)=0$.

(2) $n_{1}=1, n_{2}=2, A B C\left(T_{1} \circ T_{2}\right)=(3 \sqrt{2} / 2)$.

(3) $n_{1}=1, n_{2}=3$, $A B C\left(T_{1} \circ T_{2}\right)=A B C\left(T_{1} \circ P_{3}\right)=2 \sqrt{2}+(2 / 3)$.

(4) $n_{1}=2, n_{2}=1, A B C\left(T_{1} \circ T_{2}\right)=(3 \sqrt{2} / 2)$.

(5) $n_{1}=2, n_{2}=2, A B C\left(T_{1} \circ T_{2}\right)=3 \sqrt{2}+(2 / 3)$.

(6) $n_{1}=2, n_{2}=3, \quad A B C\left(T_{1} \circ T_{2}\right)=A B C\left(P_{2} \circ\right.$ $\left.P_{3}\right)=4 \sqrt{2}+2 \sqrt{(5 / 12)}+(\sqrt{6} / 4)$.

\section{Conclusions}

In this paper, we mainly determined the upper bounds of the $\mathrm{ABC}$ indices of $T_{1} \circ T_{2}$. It is evident that the $\mathrm{ABC}$ index of $G_{1} \circ G_{2}$ attains the maximum value if and only if both $G_{1}$ and $G_{2}$ are complete graphs. If the $A B C$ index of $G_{1}{ }^{\circ} G_{2}$ attains the minimum value, then $G_{1}$ and $G_{2}$ must be trees. We deduced that the ABC index of $T_{1} \circ T_{2}$ attains the maximum value if and only if $T_{1}$ is a path and $T_{2}$ is a star. We will discuss the minimum value of the $\mathrm{ABC}$ indices of the corona of two trees in near future.

\section{Data Availability}

The data used to support the findings of this study are available from the corresponding author upon request.

\section{Conflicts of Interest}

The authors declare that there are no conflicts of interest regarding the publication of this paper.

\section{Acknowledgments}

This study was supported by the Shanxi Province Science Foundation for Youths (no. 201901D211227).

\section{References}

[1] R. Frucht and F. Harary, "On the corona of two graphs," Aequationes Mathematicae, vol. 4, no. 3, pp. 322-325, 1970.

[2] K. C. Das, I. Gutman, and B. Furtula, "On atom-bond connectivity index," Chemical Physics Letters, vol. 511, no. 4-6, pp. 452-454, 2011.

[3] J. Chen and X. Guo, "Extreme atom-bond connectivity index of graphs," MATCH Communications in Mathematical and in Computer Chemistry, vol. 65, pp. 713-722, 2011.

[4] Y. Gao and Y. Shao, "The smallest ABC index of trees with $n$ pendent vertices," MATCH Communications in Mathematical and in Computer Chemistry, vol. 76, pp. 141-158, 2016.

[5] B. Furtula, A. Graovac, and D. Vukičević, "Atom-bond connectivity index of trees," Discrete Applied Mathematics, vol. 157, no. 13, pp. 2828-2835, 2009.

[6] L. Gan, H. Hou, and B. Liu, "Some result on atom-bond connectivity index of graphs," MATCH Communications in Mathematical and in Computer Chemistry, vol. 66, pp. 669680, 2011.

[7] R. Xing, B. Zhou, and F. Dong, "On atom-bond connectivity index of connected graphs," Discrete Applied Mathematics, vol. 159, no. 15, pp. 1617-1630, 2011.

[8] A. R. Ashrafi, T. Dehghan-Zadeh, N. Habibi, and P. E. John, "Maximum values of atom-bond connectivity index in the class of tricyclic graphs," Journal of Applied Mathematics and Computing, vol. 50, no. 1-2, pp. 511-527, 2016.

[9] X. Chen, "On extremality of ABC spectral radius of a tree," Linear Algebra and Its Applications, vol. 564, pp. 159-169, 2019.

[10] W. Lin, Z. Yan, P. Fu, and J. B. Liu, "Ordering trees by their ABC spectral radii," International Journal of Quantum Chemistry, vol. 121, no. 5, 2021.

[11] H. Bian, X. Ma, E. Vumar, and H. Yu, "The wiener-type indices of the corona of two graphs," Ars CombinatoriaWaterloo then Winnipeg, vol. 107, pp. 193-199, 2012.

[12] P. Lu and L. Xue, "Resistance distance and Kirchhoff index of two corona graphs," Ars Combinatoria-Waterloo then Winnipeg, vol. 138, pp. 323-331, 2018.

[13] I. Rezaee Abdolhosseinzadeh, F. Rahbarnia, M. Tavakoli, and A. R. Ashrafi, "Some vertex-degree-based topological indices under edge corona product," Italian Journal of Pure and Applied Mathematics, vol. 38, pp. 81-91, 2017.

[14] X. Zhang, H. Jiang, J.-B. Liu, and Z. Shao, "The cartesian product and join graphs on edge-version atom-bond connectivity and geometric arithmetic indices," Molecules, vol. 23, no. 7, p. 1731, 2018.

[15] C. McLeman and E. McNicholas, "Spectra of coronae," Linear Algebra and Its Applications, vol. 435, no. 5, pp. 998-1007, 2011.

[16] S. Wang and B. Zhou, "The signless Laplacian spectra of the corona and edge corona of two graphs," Linear and Multilinear Algebra, vol. 61, no. 2, pp. 197-204, 2013.

[17] R. Sharma, B. Adhikari, and A. Mishra, "Structural and spectral properties of corona graphs," Discrete Applied Mathematics, vol. 228, pp. 14-31, 2017.

[18] S. Klavžar and M. Tavakoli, "Dominated and dominator colorings over (edge) corona and hierarchical products," 
Applied Mathematics and Computation, vol. 390, p. 125647, 2021.

[19] W. Lin, J. Chen, Z. Wu, D. Dimitrov, and L. Huang, "Computer search for large trees with minimal ABC index," Applied Mathematics and Computation, vol. 338, pp. 221-230, 2018. 